\title{
Neoadjuvant chemoimmunotherapy in resectable stage IIIA/IIIB non-small cell lung cancer
}

\author{
Yulong Chen ${ }^{1 \#}$, Bo Yan ${ }^{2 \#}$, Feng Xu ${ }^{1 \#}$, Zhenzhen Hui ${ }^{3}$, Gang Zhao ${ }^{4}$, Jie Liu ${ }^{5}$, Huan Zhang ${ }^{6}$ Ziqing Zeng ${ }^{3}$, \\ Ran Zhang ${ }^{7}$, Mariano Provencio ${ }^{8}$, Xiubao Ren $^{3}$, Jian You ${ }^{1}$ \\ ${ }^{1}$ Department of Lung Cancer, Tianjin Lung Cancer Center, Tianjin Medical University Cancer Institute and Hospital, National Clinical \\ Research Center for Cancer, Key Laboratory of Cancer Prevention and Therapy, Tianjin's Clinical Research Center for Cancer, Tianjin, China; \\ ${ }^{2}$ Department of Radiotherapy, National Clinical Research Center of Cancer, Tianjin Medical University Cancer Institute and Hospital, Tianjin, \\ China; ${ }^{3}$ Immunology Laboratory, National Clinical Research Center of Cancer, Tianjin Medical University Cancer Institute and Hospital, Tianjin, \\ China; ${ }^{4}$ Department of Pathology, National Clinical Research Center of Cancer, Tianjin Medical University Cancer Institute and Hospital, Tianjin, \\ China; ${ }^{5}$ College of Computer and Control Engineering, College of Software, Nankai University, Tianjin, China; ${ }^{6}$ Cancer Prevention Center, \\ Tianjin Medical University Cancer Institute and Hospital, National Clinical Research Center for Cancer, Key Laboratory of Cancer Prevention and \\ Therapy, Tianjin, Tianjin's Clinical Research Center for Cancer, Tianjin, China; ${ }^{7}$ Department of Oncology Surgery, Tianjin Cancer Hospital Airport \\ Free Trade Zone Hospital Tianjin, China; ${ }^{8}$ Hospital Universitario Puerta de Hierro-Majadahonda, Madrid, Spain \\ Contributions: (I) Conception and design: J You, X Ren; (II) Administrative support: J You; (III) Provision of study materials or patients: Y Chen; \\ (IV) Collection and assembly of data: Y Chen, B Yan, Z Hui, Z Zeng; (V) Data analysis and interpretation: Y Chen, H Zhang; (VI) Manuscript \\ writing: All authors; (VII) Final approval of manuscript: All authors. \\ \#These authors contributed equally to this work. \\ Correspondence to: Jian You. Department of Lung Cancer, Tianjin Lung Cancer Center, Tianjin Medical University Cancer Institute and Hospital, \\ Huan-Hu-Xi Road, Ti-Yuan-Bei, He Xi District, Tianjin 300060, China. Email: youjiancn@126.com; Xiubao Ren. Immunology Lab, National Clinical \\ Research Center of Cancer, Tianjin Medical University Cancer Institute and Hospital, Tianjin 300060, China. Email: renxiubao@tjmuch.com.
}

Background: A small proportion of patients with non-small cell lung cancer (NSCLC) experience objective clinical benefit after neoadjuvant programmed cell death 1 (PD-1) blockade. A neoadjuvant therapeutic regimen combining immune checkpoint blockade with chemotherapy might improve the treatment effect, but such a regimen has not been tested in patients with resectable stage IIIA/IIIB NSCLC.

Methods: A retrospective study of 35 patients with resectable stage IIIA and IIIB NSCLC who were treated with neoadjuvant chemoimmunotherapy (NCIO) was performed. Patients were evaluated for pathological complete response (pCR), major pathologic response (MPR), safety, and feasibility. The correlations of pathologic response with various clinical factors were studied to identify predictors of pathological response.

Results: NCIO was associated with few immediate adverse events. NCIO did not delay planned surgery and led to a pCR rate of $51.43 \%$ and an MPR rate of $74.29 \%$ for the primary tumor. No association was observed between programmed death-ligand 1 (PD-L1) expression before NCIO and the pathologic response (Pearson's $\mathrm{r}=-0.071 ; \mathrm{P}=0.685$ ). However, a significant difference was observed in pathological response in patients with intracavitary and extracavitary tumors $(\mathrm{P}<0.05)$. Patients with intracavitary type had a higher pCR (76.47\% vs. $31.58 \%)$ and MPR (100\% vs. $50.00 \%)$ rate than patients with extracavitary type (Pearson's $r=0.7280 ; \mathrm{P}=0.0009)$.

Conclusions: NCIO was associated with few side effects, did not delay surgery, and achieved a pCR in $51.43 \%$ and MPR in $74.29 \%$ of resected tumors. No significant correlation was found between pathologic response and PD-L1 expression. While the intracavitary and extracavitary tumors type T was predictive of the pathological response to NCIO.

Keywords: Neoadjuvant chemoimmunotherapy; non-small cell lung cancer (NSCLC); programmed cell death 1 (PD-1); surgery 
Submitted Jan 20, 2021. Accepted for publication May 21, 2021.

doi: $10.21037 /$ tlcr-21-329

View this article at: http://dx.doi.org/10.21037/tlcr-21-329

\section{Introduction}

The majority of the treatment methods have focused on the establishment of new treatment options for non-small cell lung cancer (NSCLC). Among various immunotherapies, programmed cell death 1 (PD-1) and programmed cell death-ligand 1 (PD-L1) immune checkpoint inhibitors (ICIs) have received the most attention so far (1). Therapeutic blocking of the PD-1/PD-L1 axis using specific targeted monoclonal antibodies activates the immune system to recognize and target cancer cells via a $\mathrm{T}$ cell-mediated immune response (2). Recently, neoadjuvant PD-1 blockade was also investigated in resectable NSCLC, but only a small percentage of patients obtained pathologic complete response (pCR). The clinical trial LCMC3 (ClinicalTrials.gov numbers: NCT02927301) reported that the MPR rate in the initial 82 patients was $18 \%$ with pCR rate of $4 \%$ (3). Thus, the combination of checkpoint blockade and chemotherapy possesses the potential for synergistic immune activation. Several clinical studies have focused on neoadjuvant immunotherapy prior to tumor resection for advanced NSCLC. At the 2019 World Conference on Lung Cancer, it was reported that neoadjuvant anti-PD-1 immunotherapy did not delay surgery and achieved an MPR in $83 \%$ of patients who underwent tumor resection in a clinical study (4). However, during the 2020 ASCO Annual Meeting, Zinner reported that only $6(46 \%)$ and $5(38 \%)$ patients out of a group of 13 patients with resectable NSCLC achieved MPR and pCR, respectively (5). Recently, Shu reported on a phase II clinical trial of neoadjuvant atezolizumab and chemotherapy in patients with resectable NSCLC. Out of 30 patients, there were 17 (57\%; 95\% CI: 37-75\%) cases of MPR and $10(33 \% ; 17-53 \%)$ cases of pCR (6). And there are several case reports related the neoadjuvant immunotherapy prior to tumor resection for advanced NSCLC $(7,8)$. However, a systematic and in-depth discussion of this topic has yet to be reported in any journal, and a high-performance predictive factor, which would be critical and of tremendous assistance to avoiding non-essential surgery, still needs to be identified. Herein, we examined the safety and feasibility of the use of neoadjuvant PD-1 antibody plus chemotherapy in a small group of patients with resectable stage IIIA/IIIB NSCLC. In addition, the correlations of pathologic response with various clinical factors, including PD-L1 expression, CD4+ and CD8+ T cells, regulatory T cells (Tregs), and intracavitary or extracavitary type, were analyzed to identify predictors of pathological response. We present the following article in accordance with the STROBE reporting checklist (available at http://dx.doi. org/10.21037/tlcr-21-329).

\section{Methods}

\section{Patients}

A retrospective study of 35 patients with resectable stage IIIA and IIIB NSCLC who were treated with pembrolizumab plus chemotherapy as neoadjuvant chemoimmunotherapy in Tianjin Medical University Cancer Institute and Hospital (TJMUCH; Tianjin, China) was conducted. Clinical staging was done according to the American Joint Committee on Cancer (AJCC) $8^{\text {th }}$ edition.

The therapeutic safety and feasibility were studied, together radiological and pathological responses to treatment. Adverse events were classified according to the National Cancer Institute Common Terminology Criteria for Adverse Events, version 4.0. Changes in the size of tumors were evaluated according to the Response Evaluation Criteria in Solid Tumors (RECIST) version 1.1 (9). All patients underwent $\mathrm{R} 0$ resection including lobectomy, sleeve lobectomy, complete pneumonectomy, pulmonary arterioplasty, and radical dissection of lymph nodes. Feasibility was defined as any delay in the planned surgery of $<30$ days. All procedures performed in this study involving human participants were in accordance with the Declaration of Helsinki (as revised in 2013). The study was approved by the institutional review board of TJMUCH (NO.: bc2020060) and informed consent was taken from all the patients.

\section{Immunobistochemistry}

PD-L1 was analyzed by immunohistochemistry (IHC) in the Pathology Department of TJMUCH using the Monoclonal Mouse Anti-Human PD-L1 clone 22C3 (LOT10145059, Dako, Carpentaria, CA, USA). The tumor proportion score (TPS) and combined positivity score (CPS) 
were calculated as the percentage of at least 100 viable cells with complete or partial membrane staining. In patients with no residual tumor after the treatment, only the CPS was calculated. Interpretation of TPS and CPS of patients was provided by a pathologist at TJMUCH.

\section{Flow cytometry}

Fresh tumor tissues obtained during surgery were mechanically and enzymatically disaggregated into a single cell suspension. CD45, CD3, CD4, CD8, CD127, and CD25 were purchased from BioLegend (San Diego, CA, USA). The following antibodies were used: FITC antihuman CD4 317408; PE anti-human CD25 302606; PE anti-human CD163 326506; PerCP/Cy5.5 anti-human CD127 (IL-7R $\alpha$ ) 351322; APC anti-human CD68 333810; Brilliant Violet 421 anti-human CD3 300434; and Brilliant Violet 510 anti-human CD45 304036. Cells were stained with optimal amounts of each antibody for 30 minutes at $4{ }^{\circ} \mathrm{C}$ in staining buffer, according to the manufacturer's protocol. Data (10,000 events) were acquired on a BD-Aria II flow cytometer (BD Biosciences, San Diego, CA, USA) and analyzed using FlowJo software (FlowJo LLC, Ashland, OR, USA).

\section{Pathological assessment}

Pathological response was assessed by local pathologists, who measured the percentage of residual viable tumor in primary tumors resected from each patient during surgery using previously reported methods $(1,6,10,11)$. All tumor bed samples measuring less than $6 \mathrm{~cm}$ at their greatest diameter were submitted in their entirety. For tumor bed samples with a greatest diameter of $6 \mathrm{~cm}$ or more, a minimum of 1 section per $\mathrm{cm}$ of the greatest dimension of the tumor bed was assessed. Tumor tissue samples were sectioned, and the percentage of viable tumor tissue was recorded for each tumor slide. The average percentage of viable tumor tissue for each patient was then calculated. Tumors with $<10 \%$ viable tumor cells were considered to have an MPR, and those with no viable tumor cells were deemed to be pCR.

\section{Definition of intracavitary and extracavitary tumors type}

Intracavitary and extracavitary tumors type are defined as follows. For the intracavitary type, bronchoscopy shows protrusive growth of intrabronchial masses and obstruction of the bronchus. The CT image shows distal fan-shaped atelectasis. And for the extracavitary type, bronchoscope shows bronchial mucosa redness, swelling, shrinkage, and the CT image only shows central bronchial masses (Figure S1).

\section{Statistical analysis}

The Mann-Whitney U test was used to compare pathological response between patients grouped by invasion and non-invasion status and TPS score. The independentsamples $t$-test was used to compare the proportions of CD4, CD8, and Tregs between genotype cohorts. Progressionfree survival (PFS) and overall survival (OS) were estimated using Kaplan-Meier curves and the log-rank test. Pearson's correlation coefficient was used to analyze the correlation between TPS score and pathological response. All P-values were based on a 2-sided hypothesis, and data were analyzed using SPSS 22.0 (IBM Corporation, New York, USA).

\section{Results}

\section{Patient characteristics}

From January 2019 to May 2020, 35 patients with resectable stage IIIA or IIIB non-NSCLC received neoadjuvant chemoimmunotherapy at TJMUCH. The median age of the cohort was $62.17 \pm 5.99(43-72)$ years. Of the patients, $82.86 \%$ were male, $74.28 \%$ had squamous carcinoma, $88.57 \%$ were stage IIIA, and $57.14 \%$ were PD-L1 positive $(\geq 50 \%)$. For the clinical TNM stage, 11 patients were T1N2M0, 8 were T2N2M0, 11 were T3N1M0, 4 were T3N2M0, and 1 was T4N1M0. All the patients underwent baseline tumor staging, including pretreatment biopsy, pathological evaluation of mediastinal lymph nodes (if indicated) by bronchoscopy or mediastinoscopy, positronemission tomography-computed tomography (PET-CT), and contrast-enhanced CT or magnetic resonance imaging of the brain and chest. Patients with squamous carcinoma received 2 courses of pembrolizumab $2 \mathrm{mg} / \mathrm{kg}$ intravenous (IV) $\mathrm{q} 3 \mathrm{w}$ added to cisplatin $75 \mathrm{mg} / \mathrm{m}^{2} \mathrm{IV} \mathrm{q} 3 \mathrm{w}$ plus paclitaxel liposome $135 \mathrm{mg} / \mathrm{m}^{2} \mathrm{q} 3 \mathrm{w}$; patients with non-squamous carcinoma received pemetrexed $500 \mathrm{mg} / \mathrm{m}^{2} \mathrm{IV}$ q3w instead of paclitaxel liposome (Table 1).

\section{Safety and feasibility}

Neither neoadjuvant pembrolizumab plus paclitaxel liposome nor pemetrexed combined with cisplatin resulted 
Table 1 Characteristics of the patients at baseline according to the pathological response

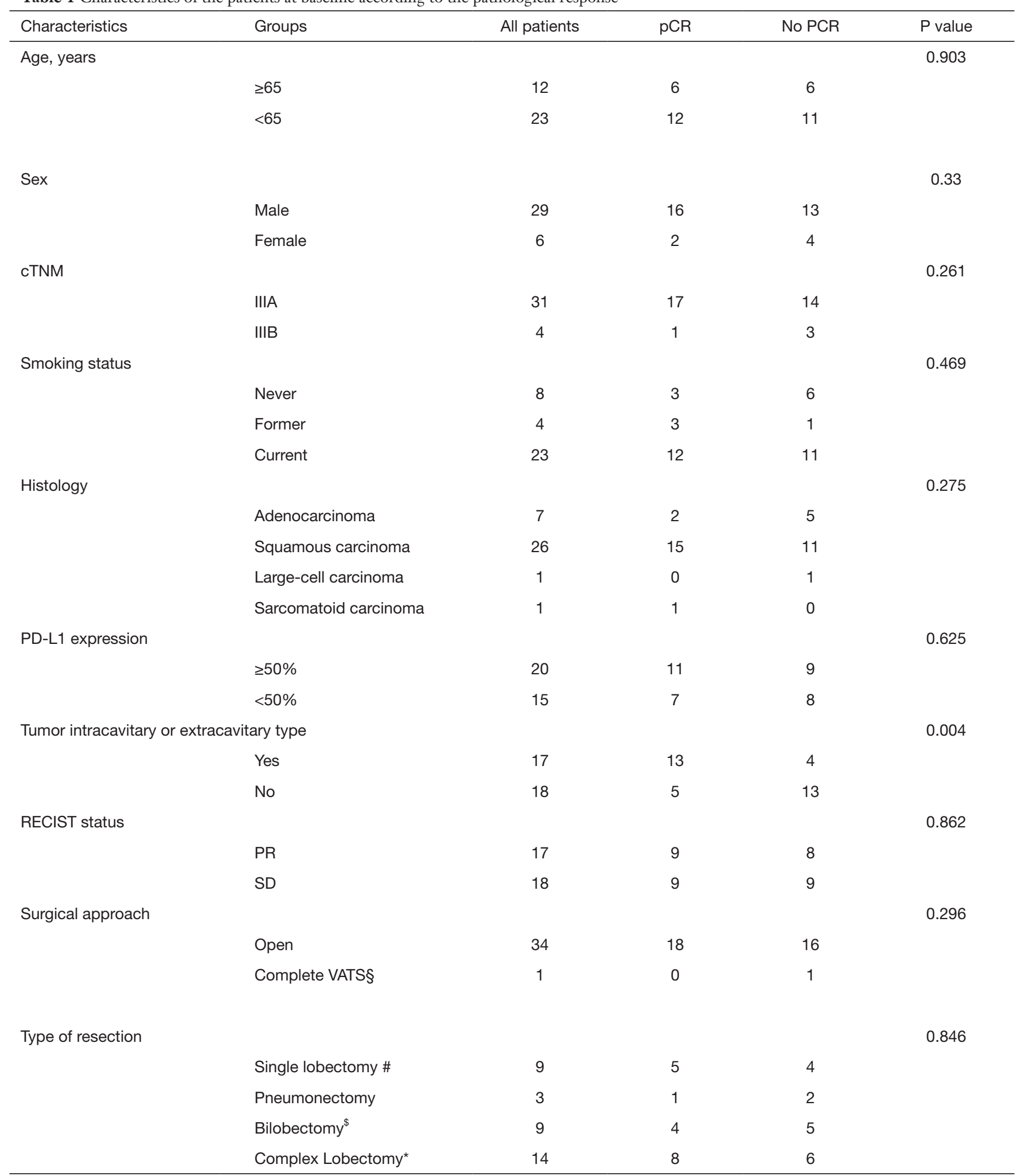

\footnotetext{
§, video-assisted thoracoscopic surgery; ${ }^{\prime}$, does not include pneumonectomy, bilobectomy, or complex lobectomy; ${ }^{\$}$, does not include pneumonectomy; *, complex lobectomy including sleeve resection and sleeve or partial resection of the pulmonary artery or surgery for pancoast tumors.
} 


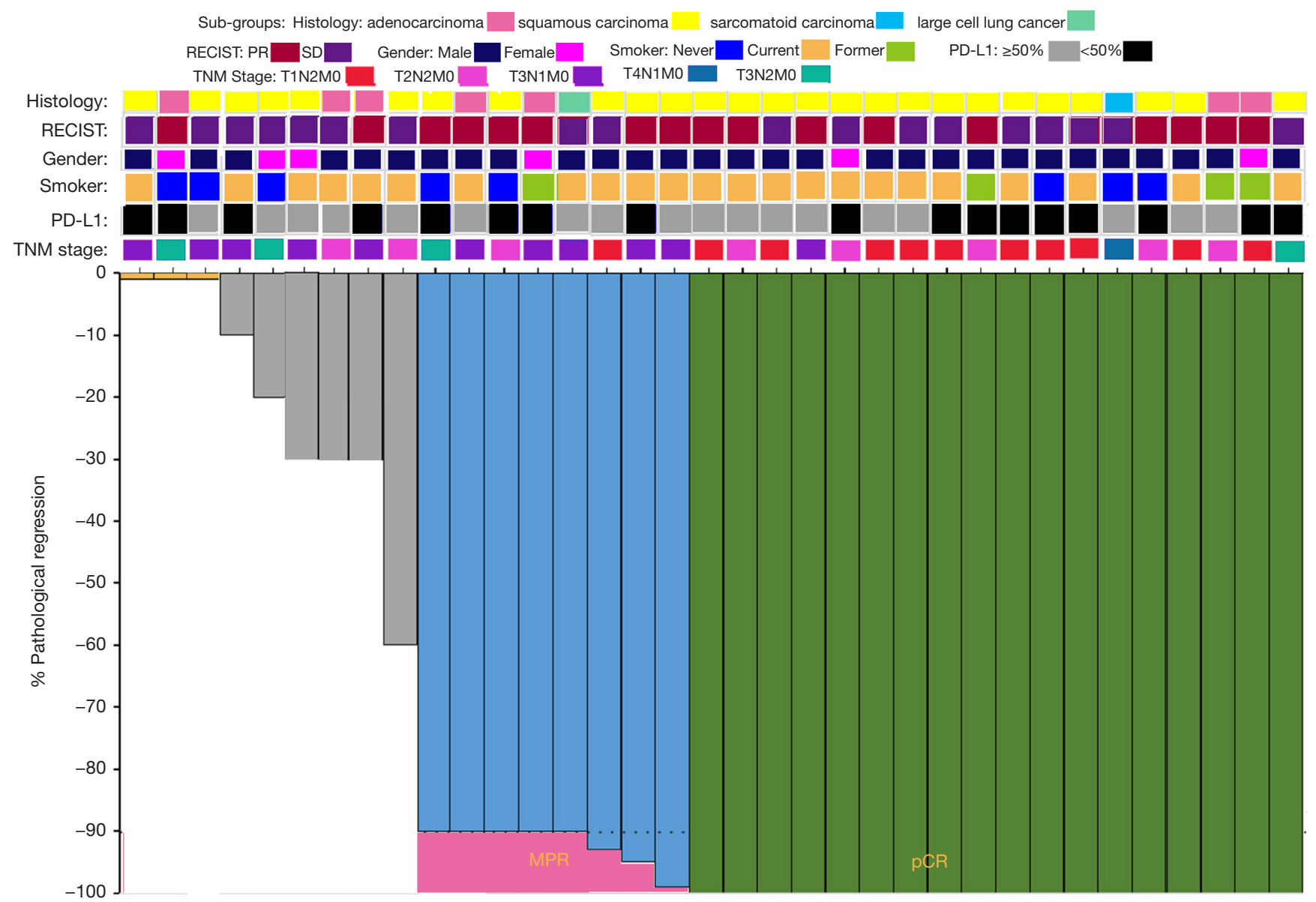

Figure 1 Pathological assessment of response to NCIO. Pathological response in the resected primary lung tumor after NCIO in each of the 35 patients who underwent surgical resection, according to the percentage of remaining viable tumor cells. The gray horizontal line indicates the threshold for a major pathological response (90\% regression). Clinical and pathological features including histology, preoperative radiologic response (according to RECIST), sex, smoking status, PD-L1 expression in the pretreatment biopsy sample, and TNM stage are annotated for each patient. PR, partial response; SD, stable disease, PD-L1, programmed death-ligand 1.

in any previously unreported toxic effects. Before surgery, 1 patient developed a grade 3 toxicity (rash). All 35 eligible patients $(100 \%)$ underwent complete tumor resection. The median interval between the administration of the second dose of NICO O and surgery was 33.4 (range, 28-35) days. No treatment-related surgical delays occurred as defined in the protocol. Of the 35 patients enrolled, 18 (51.43\%) patients achieved pCR, and 26/35 (74.29\%) patients exhibited MPR. Also, 1 patient had no residual tumor in the primary tumor but had residual tumor in hilar lymph nodes, which we considered as $0 \%$ pathological regression. According to their pathological responses, the patients were divided into a pCR group and a non-pCR group. In the pCR group, the median age was $61.5 \pm 6.88$ years. Of the 18
pCR patients, $88.89 \%$ were male, $83.33 \%$ had squamous carcinoma, $94.4 \%$ were stage IIIA, and $61.10 \%$ had a PDL1 TPS $>50 \%$. The non-pCR group had a median age of $62.88 \pm 5.01$ years. Of the 17 non-PCR patients, $76.47 \%$ were male, $64.71 \%$ had squamous carcinoma, $82.35 \%$ were stage IIIA, and $52.94 \%$ had a PD-L1 TPS $>50 \%$ (Table 1 and Figure 1).

After a median of 13.29 (range, 3-24) months of postoperative follow-up, $94.29 \%$ (33/35) of patients who had undergone surgical resection were alive and recurrence-free. One patient died 3 months after surgery because of cerebral ischemic stroke (patient 3), and another died 10 months after surgery due to mass N2 lymph node metastasis (patient 1). One patient was diagnosed with brain metastasis (patient 25) 

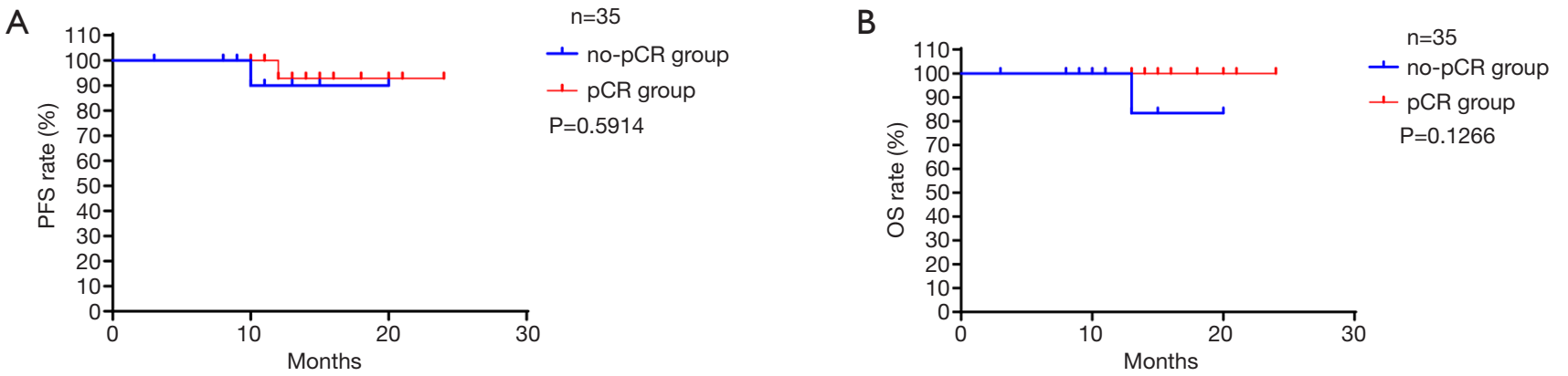

Figure 2 Kaplan-Meier curves of PFS and OS for the pCR and non-pCR groups. PFS curve (A) and OS curve (B) for all patients. OS, overall survival; pCR, pathologic complete response; PFS, progression-free survival.

12 months after the surgery. The median duration of recurrence-free survival had not been reached at the time of data analysis. Hence, based on the available data, no significant differences were detected in PFS and OS between the pCR and non-pCR groups (Figure 2).

\section{Patbological findings after neoadjuvant PD-1 blockade}

Representative radiological and pathological responses before and after 2 cycles of NCIO are shown in Figure $3 \mathrm{~A}$. MPR occurred in 26/35 (74.29\%) patients, and 18 patients $(51.43 \%)$ achieved a pCR in the primary tumor. One patient exhibited pCR despite apparent tumor enlargement on preoperative CT (possibly due to infiltration of immune cells into the tumor), which was defined as pseudoprogression. In primary tumors with MPR, we observed a large number of infiltrating lymphocytes and macrophages. This finding was compatible with an immunological mechanism of response as well as the phenomenon of necrotic tumor being associated with fibrotic tissue repair (Figure $3 A$ ).

\section{Correlation of intracavitary and extracavitary tumors type with pathological response}

In all the patients, we also analyzed intracavitary and extracavitary tumors type by bronchoscopy based on CT. $17 / 35(48.57 \%)$ patients were evaluated as intracavitary and $18 / 35$ (51.43\%) as extracavitary. Thirteen (76.47\%) patients in the invasion group achieved pCR, and all 17 (100\%) patients showed an MPR. Meanwhile, in the non-invasion group, only $5(27.78 \%)$ patients showed a pCR, and $9(50.00 \%)$ patients did not obtain an MPR. The Pearson's rho was 0.7280 , and there was a significant difference between the 2 groups $(\mathrm{P}=0.0009 ;$ Figure 3B).

\section{Expression of PD-L1 with pathological response}

The expression of PD-L1 could be evaluated in pretreatment biopsy samples in all patients (Figure $3 C$ ). A pCR or MPR was found to have occurred in PD-L1-positive and PDL1-negative tumors. The correlation between the TPS of pretreatment biopsy samples and pathological regression was analyzed. As shown in Figure $3 C$, there was no association between PD-L1 expression before treatment and the pathological response to NCIO (Pearson's $r=-0.071$; $\mathrm{P}=0.685$ ).

\section{Immune proofing of T cells to NCIO}

To further explore the T-cell changes before and after NCIO of these patients, flow cytometry analysis was performed. CD4+ and CD8+ T cells and Tregs were evaluated in post-surgical samples available from 17 patients (Figure 3D). The ratios of CD4+ T cells, CD8+ T cells, and Tregs to $\mathrm{T}$ cells and all cells in the sample were analyzed in pCR and MPR patients. As shown in Figure 3D, in the pCR group and non-PCR group, the proportion of CD $4+\mathrm{T}$ cells to $\mathrm{T}$ cells was $36.93 \pm 12.34$ and $43.67 \pm 14.61$, respectively $(\mathrm{P}>0.05)$, and the proportion of $\mathrm{CD} 4+\mathrm{T}$ cells to all cells in the sample was $7.42 \pm 4.94$ and $10.39 \pm 11.90$, respectively $(\mathrm{P}>0.05)$. Furthermore, the proportion of CD8+ T cells to $\mathrm{T}$ cells in the pCR and non-PCR groups was $50.74 \pm 12.78$ and $43.90 \pm 19.70$, respectively $(\mathrm{P}>0.05)$, and that of $\mathrm{CD} 8+\mathrm{T}$ cells to all cells in the sample was $10.43 \pm 7.97$ and $9.71 \pm 6.97$, respectively $(\mathrm{P}>0.05)$. The proportion of Tregs to $\mathrm{T}$ cells in the pCR and non-PCR groups was $1.55 \pm 1.25$ and $3.57 \pm 2.69$, respectively $(\mathrm{P}>0.05)$, 
A

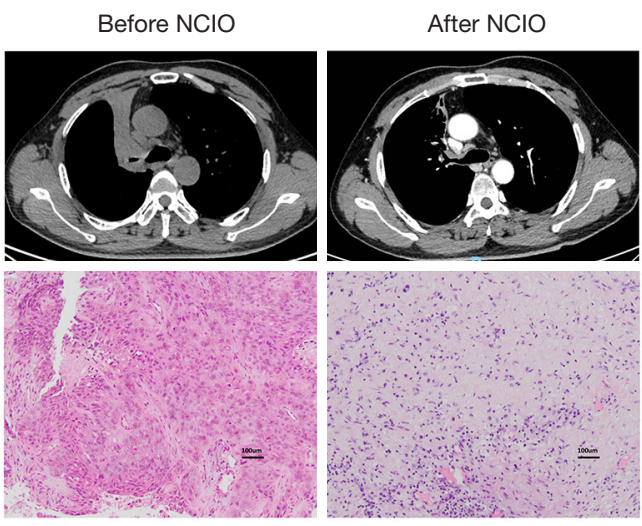

Patient 28
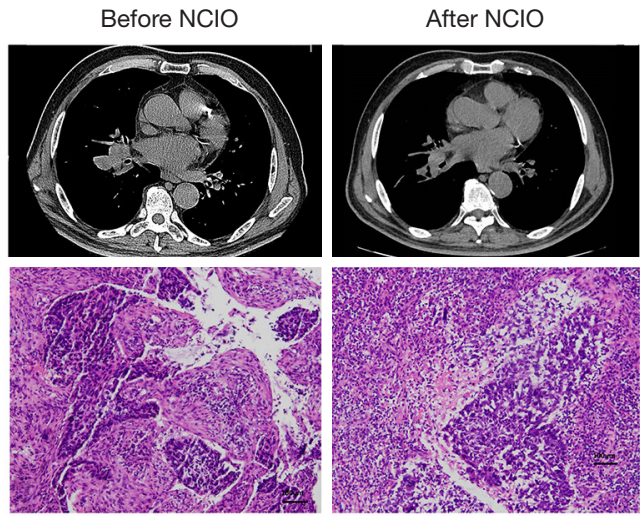

Patient 9

B

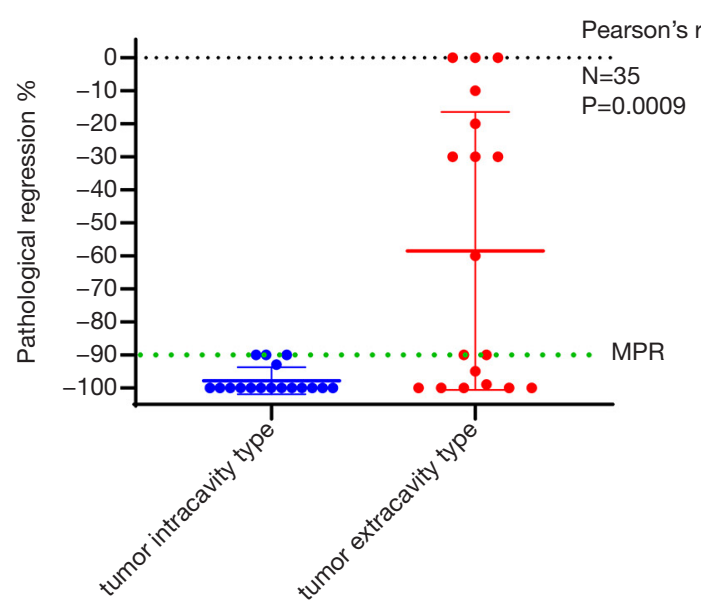

C

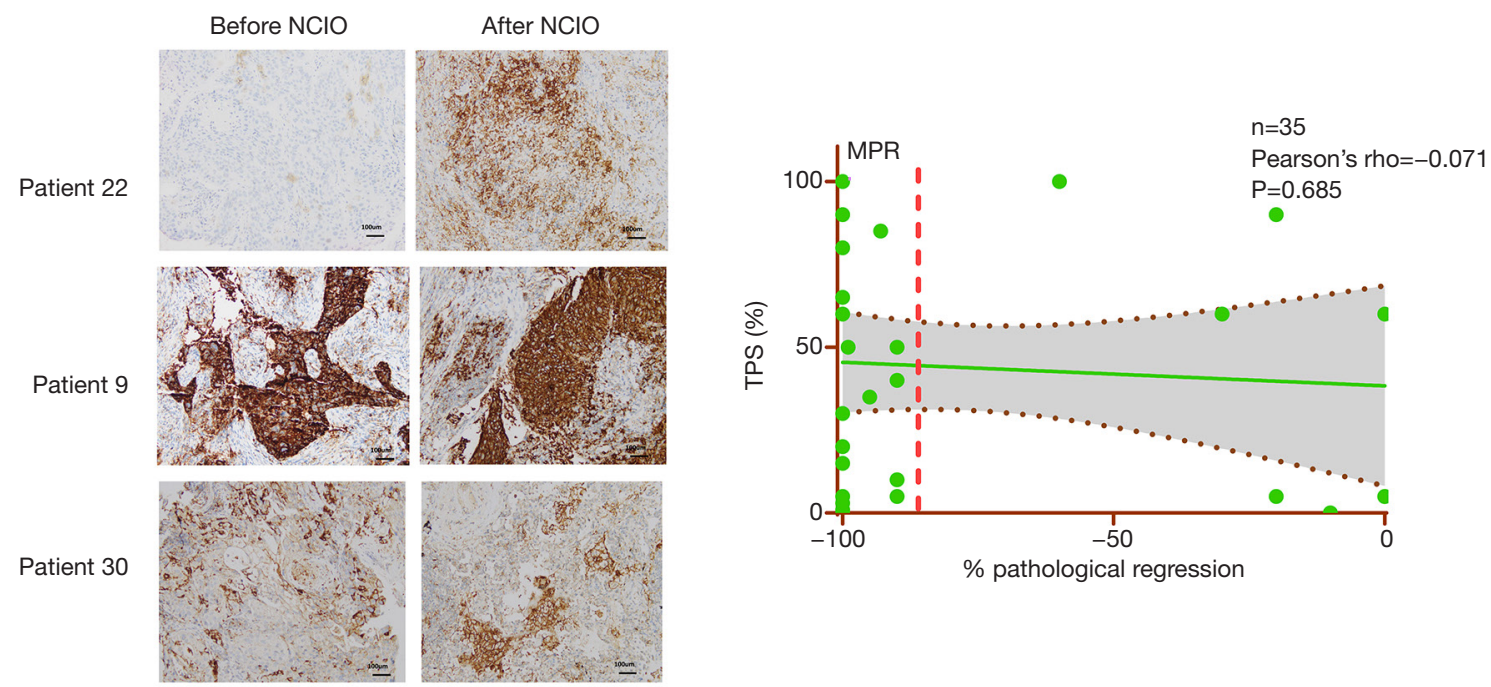




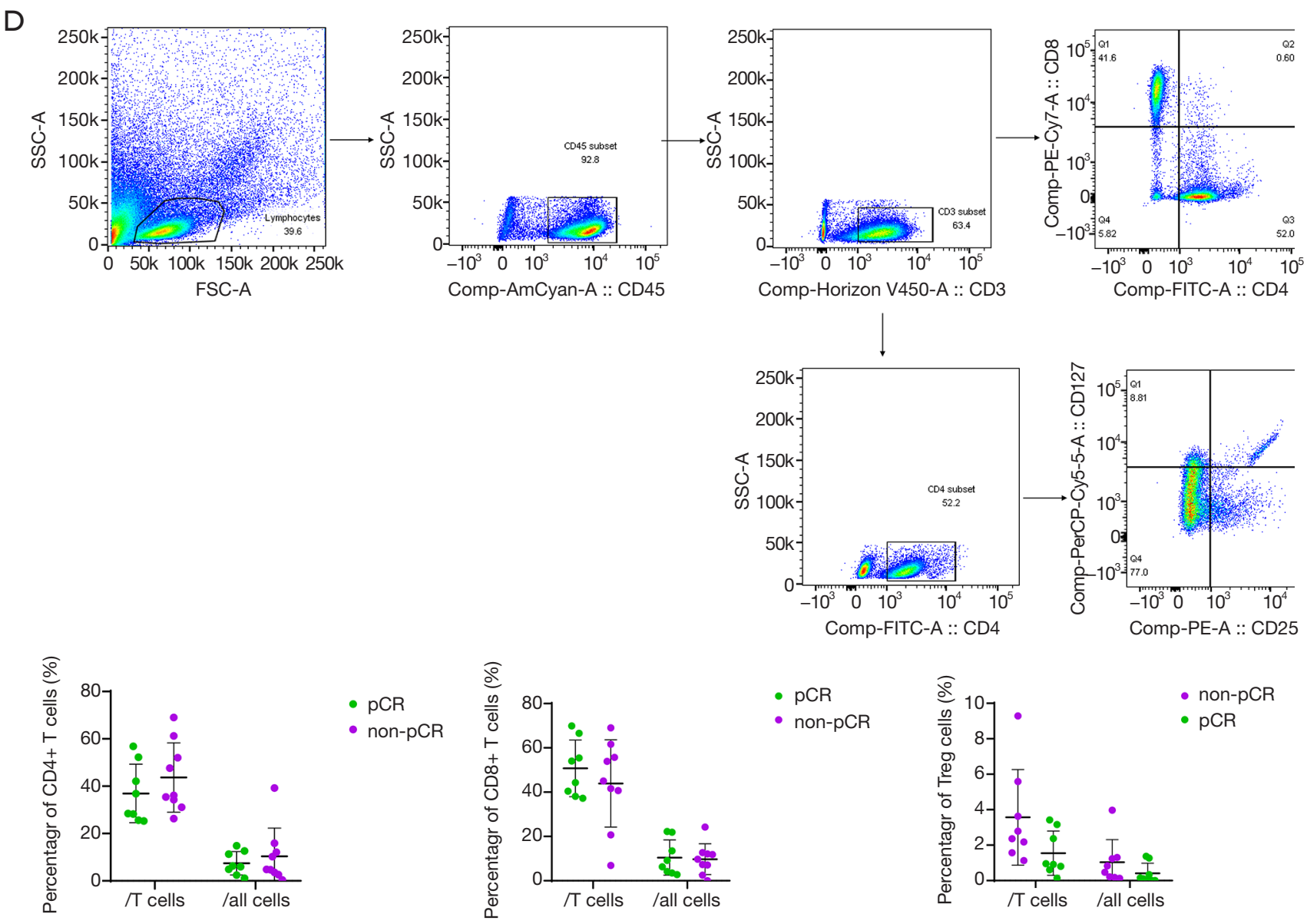

Figure 3 Characteristic changes before and after NCIO. (A) Patterns of radiological and pathological response to NCIO. Left: CT imaging of the chest of a 56-year-old male never-smoker (patient 28) with stage IIIA squamous lung cancer before and after the administration of NCIO. In the upper panel, the pretreatment scan shows a primary tumor mass with pulmonary atelectasis in the upper lobe of the right lung. A scan performed before the surgery shows $90 \%$ shrinkage with associated tumor cavitation. The lower panel consists of the representative sections of tumor specimens obtained from the patient before the administration of NCIO and after (right) HE staining. This patient had $100 \%$ pathological regression of the large primary lung tumor and no lymph-node metastases in the resected specimen. Right: CT of the chest of a 63-year-old male smoker (patient 9) with stage IIIA lung adenocarcinoma. In the upper panel, the tumor appears stable on imaging before and after NCIO. In the lower panel, representative sections of tumor specimens obtained from the patient before (left) and after (right) HE. Neoplastic cells are present throughout the pre-treatment specimen, whereas in the post-treatment specimen, $60 \%$ tumor regression was detected. (B) Association between intracavitary and extracavitary tumors type e and pathological response to NCIO. The association between intracavitary and extracavitary tumors type and pathological response to the NCIO was analyzed. Patients with intracavitary type had higher pCR and MPR rates as compared with those with extracavitary type (pCR rate: $76.47 \%$ vs. $31.58 \%$; MPR rate: $100 \%$ vs. $50.00 \%$ ). The Pearson's rho is 0.7280 , and a significant difference can be seen between the 2 groups $(\mathrm{P}=0.0009)$. (C) Association of PD-L1 with pathological response to NCIO. Left: PD-L1 expression changes before and after NCIO. Pretreatment biopsy and post-surgery samples were obtained, and PD-L1 testing was performed using the Monoclonal Mouse Anti-Human PD-L1 clone 22C3. In the upper panel, the pre-NCIO TPS of patient 26 , who had $100 \%$ pathological regression, was $0 \%$, and the post-NCIO CPS was $60 \%$. In the middle panel, the pre- and post-NCIO TPSs of patient 5 , who had $60 \%$ pathological regression, were both $100 \%$. In the lower panel, the pre-NCIO TPS of patient 30 , who had $100 \%$ pathological regression, was 30\%, and the post-NCIO CPS was 50\%. Right. No association was observed between pre-treatment PDL1 expression and pathological response to NCIO (Pearson's $\mathrm{r}=-0.071 ; \mathrm{P}=0.685$ ). The dashed black line indicates the linear regression line, and the dashed gray lines indicate the upper and lower boundaries of the $95 \%$ confidence interval. (D) Immune proofing of T cells to NCIO in the post-surgery sample. Left: Gating scheme for CD4+ and CD8+ T-cell and Treg cell intracellular cytokine cytometry. FSC-A, forward scatter A; SS, side scatter. Black polygons indicate gated cell subsets. Right: The proportions of CD4+ cells, CD8+ T-cells, and Treg cells to T cells and all cells in the sample. No significant difference was observed between the pCR group and the non-pCR group $(\mathrm{P}>0.05)$. 
and that of Tregs to all cells in the sample was $0.41 \pm 0.57$ and $1.04 \pm 1.27$, respectively $(\mathrm{P}>0.05)$.

\section{Discussion}

As we all knows that single chemotherapy or immune therapy in NSCLC is with lower effective rate and low survival rate; the pCR rate and MPR rate are very low (1-3). The combination of checkpoint blockade and chemotherapy explores the potential for synergistic immune activation and was found to be impressive in the present study. And several clinical studies have reported that neoadjuvant anti-PD-1 immunotherapy achieves an MPR in $46-83 \%$ and a pCR in $38-56 \%$ of patients who undergo tumor resection (3-8). Due to these impressive results, the number of stage IIIA or IIIB NSCLC patients in China receiving neoadjuvant chemoimmunotherapy before surgery. According to the related reports and our own experience, patients with squamous carcinoma appear to show a better pathological response than adenocarcinoma or other kind of NSCLC. Therefore, there are many more patients with squamous carcinoma than with adenocarcinoma.

Neoadjuvant therapy allows for earlier treatment of micrometastatic disease, reductions in surgical risk, improved tolerability and compliance with the therapy. In our study, we observed that neoadjuvant administration of 2 doses of pembrolizumab plus paclitaxel liposome or pemetrexed combined with cisplatin in patients with stage IIIA-IIIB resectable stage NSCLC was associated with few immediate adverse events. NCIO did not delay the planned surgery and led to a pCR in $51.43 \%$ of patients and an MPR in $74.29 \%$ of patients for the primary tumor. Interestingly, 2 patients had no residual tumor in the primary tumor but had residual tumor in hilar lymph nodes. Moreover, the treatment was not associated with any previously unreported toxic effects $(1-4,12)$. The NADIM study reported that $13 \%$ of patients encountered grade 3-5 treatment-related adverse events, with the most common postsurgical complication being respiratory infection (12). In the current study, only 1 patient encountered an adverse event of grade $3-5$ in the form of grade 3 rash, and none of the patients experienced postsurgical complications such as respiratory infections.

Immune-checkpoint blockade can yield long-term survival benefits for a subset of cancer patients $(12,13)$. Specifically, only patients with advanced solid tumors show a response to this treatment. Therefore, developing a method to identify the patients who are most likely to respond to immunotherapy is essential. Several predictors, such as PD-
L1 (14-16), tumor mutational burden (17), and radiomics (18), have been explored to help in the prediction of clinical outcomes. However, currently, there is no reliable predictor to aid in clinical decision-making. In the present study, no significant correlation could be established between PD-L1 expression and pathological response n. Also, pathological regression did not differ significantly between patients with TPS scores $\geq 50 \%$ and $<50 \%$ (Pearson's $r=-0.071$ and $\mathrm{P}=0.685$ ), with cases of $\mathrm{pCR}$ and MPR occurring in patients with high and low TPS. PD-L1 expression is not a good predictor of pathological response. Hence, we studied the dynamic changes in squamous cell carcinoma, including changes in tumor size before and after NCIO, original tumor volume, carcinoembryonic antigen (CEA) and squamous cell carcinoma antigen (SCC) in response to NCIO. These factors did not show any significant difference between patients with and without pCR. Surprisingly, intracavitary and extracavitary tumors type $\mathrm{T}$ was associated with pathological response. Of the patients in the invasion group, $76.47 \%$ showed pCR and $100 \%$ exhibited MPR, compared to only $31.58 \%$ and $50 \%$ of patients, respectively, in the non-invasion group $(\mathrm{P}<0.001)$. Furthermore, the Pearson's rho was 0.7280 , which indicated that intracavitary and extracavitary tumors types might be a valuable parameter for the prediction of the pathological response to NCIO.

It is thought that PD-1 blockade has the highest likelihood of success in tumors microenvironment with functional CD8+ TILs, functional antigen presentation machinery proteins, and T-helper type 1 cytokines and chemokines such as IFN- $\gamma$ and IL-2 (19-21). The PD-1 blockade is difficult to play a role if the tumor is completely deprived of immune cells (immune desert), or the immune cells are unable to infiltrate the tumor properly (immune-excluded tumors). Combination therapy with immunotherapy and chemotherapy may solve the problem. Recent data show that chemotherapy is also possess immunostimulatory properties. It has the potential to induce favorable immunogenic conditions within the tumor microenvironment, which may be difficult to achieve by just targeting immune cells $(22,23)$. Furthermore, we also studied the immune proofing of T cells to the NCIO and identified CD4+ and CD8+ T cells and Tregs. Goldberg et al. demonstrated that PD-1 blockade enhances earlystage T-cell activation in lymph nodes (24). Liu et al. reported that neoadjuvant $\mathrm{PD}-1$ blockade enhances the systemic priming of antitumor $\mathrm{T}$ cells, thereby potentially eliminating micrometastatic cancer cells that might otherwise cause postsurgical relapse (25). Our findings did 
not reveal any significant differences in CD4+ and CD8+ $\mathrm{T}$ cells and Tregs between the pCR and non-pCR groups or the MPR and non-MPR groups. The number of CD4+ and CD8+ $T$ cells and Tregs was $m$ the same between the pCR and non-pCR groups. Although no difference in the number of these $T$ cells, the function of these cells might be different. Salmon et al. described the importance of dendritic cells to the antitumor effects of PD-1 pathway blockade and suggested that PD-1 blockade not only works to directly unleash intratumoral T-cell killing but also enhances the tumor antigen-driven priming of $\mathrm{T}$ cells (26). In the present study, changes in TPS before and after treatment were also analyzed. In the pCR group, the TPS increased and decreased in $64.28 \%$ and $27.27 \%$ of patients, respectively, while in the non-pCR group, 2 and 3 out of 11 patients showed an increase and decrease in TPS, respectively. Strikingly, in the pCR group, no residual tumor was left but a large number of PD-L1-positive immune cells were detected. Further investigation of the influence of anti-PD-1 drugs on cells with PD-L1 antigen might be the key to understanding the antitumor mechanism.

The present study has some limitations that need to be discussed. First, there was a small sample size and short postoperative follow-up. However, for the first time, this report has demonstrated the safety of surgical resection after treatment with pembrolizumab plus paclitaxel liposome or pemetrexed combined with cisplatin for patients with stage IIIA-IIIB NSCLC. This study is also the first to confirm the intracavitary and extracavitary tumors type as a predictor of the pathological response to NCIO, which will be critical and of tremendous assistance to avoiding non-essential surgery. Long-term follow-up study will be necessary to establish whether or not MPR and pCR translate to prolonged OS and PFS.

\section{Conclusions}

Neoadjuvant PD-1 blockade plus chemotherapy was associated with few side effects, did not delay surgery, and induced a pCR in $51.43 \%$ of resected tumors. The results of the current study support the widespread use of NCIO for resectable stage IIIA and IIIB NSCLC patients. No significant correlation was found between pathological response and PD-L1 expression, but the intracavitary and extracavitary tumors type was predictive of the pathological response to NCIO. Studies with a longer follow-up will be necessary to investigate whether MPR and pCR are associated with improved OS and PFS.

\section{Acknowledgments}

We thank the patients, their families, and caregivers for participating in this study. We also thank You Zhou (Merck) for collaboration regarding safety analyses, and Lianmin Zhang, Hui Chen, and Meng Lu for performing the surgeries after the treatment. Medical writing assistance was provided by MedSci company. The authors also appreciate the academic support from AME Lung Cancer Collaborative Group.

Funding: None.

\section{Footnote}

Reporting Checklist: The authors have completed the STROBE reporting checklist. Available at http://dx.doi. org/10.21037/tlcr-21-329

Data Sharing Statement: Available at http://dx.doi. org/10.21037/tlcr-21-329

Conflicts of Interest: All authors have completed the ICMJE uniform disclosure form (available at http://dx.doi. org/10.21037/tlcr-21-329). The authors have no conflicts of interest to declare.

Ethical Statement: The authors are accountable for all aspects of the work in ensuring that questions related to the accuracy or integrity of any part of the work are appropriately investigated and resolved. All procedures performed in this study involving human participants were in accordance with the Declaration of Helsinki (as revised in 2013). The study was approved by the institutional review board of TJMUCH (NO.: bc2020060) and informed consent was taken from all the patients.

Open Access Statement: This is an Open Access article distributed in accordance with the Creative Commons Attribution-NonCommercial-NoDerivs 4.0 International License (CC BY-NC-ND 4.0), which permits the noncommercial replication and distribution of the article with the strict proviso that no changes or edits are made and the original work is properly cited (including links to both the formal publication through the relevant DOI and the license). See: https://creativecommons.org/licenses/by-nc-nd/4.0/. 


\section{References}

1. Forde PM, Chaft JE, Smith KN, et al. Neoadjuvant PD-1 blockade in resectable lung cancer. $\mathrm{N}$ Engl J Med 2018;378:1976-86.

2. Brahmer JR, Pardoll DM. Immune checkpoint inhibitors: making immunotherapy a reality for the treatment of lung cancer. Cancer Immunol Res 2013;1:85-91.

3. Kwiatkowski DJ, Rusch VW, Chaft JE, et al. Neoadjuvant atezolizumab in resectable non-small cell lung cancer (NSCLC): Interim analysis and biomarker data from a multicenter study (LCMC3). J Clin Oncol 2018;36:8541.

4. Provencio M, Nadal E, Insa A, et al. Neoadjuvant chemotherapy and nivolumab in resectable non-small-cell lung cancer (NADIM): an open-label, multicentre, singlearm, phase 2 trial. Lancet Oncol 2020;21:1413-22.

5. Zinner R, Axelrod R, Solomides CC, et al. Neoadjuvant nivolumab $(\mathrm{N})$ plus cisplatin $(\mathrm{C}) /$ pemetrexed $(\mathrm{P})$ or cisplatin /gemcitabine $(\mathrm{G})$ in resectable NSCLC. J Clin Oncol 2020;38:9051.

6. Shu CA, Gainor JF, Awad MM, et al. Neoadjuvant atezolizumab and chemotherapy in patients with resectable non-small-cell lung cancer: an open-label, multicentre, single-arm, phase 2 trial. Lancet Oncol 2020;21:786-95.

7. Liang H, Deng H, Liang W, et al. Perioperative chemoimmunotherapy in a patient with stage IIIB nonsmall cell lung cancer. Ann Transl Med 2020;8:245.

8. Duan H, Wang T, Luo Z, et al. Neoadjuvant programmed cell death protein 1 inhibitors combined with chemotherapy in resectable non-small cell lung cancer: an open-label, multicenter, single-arm study. Transl Lung Cancer Res 2021;10:1020-8.

9. Eisenhauer EA, Therasse P, Bogaerts J, et al. New response evaluation criteria in solid tumours: revised RECIST guideline (version 1.1). Eur J Cancer 2009;45:228-47.

10. Pataer A, Kalhor N, Correa AM, et al. Histopathologic response criteria predict survival of patients with resected lung cancer after neoadjuvant chemotherapy. J Thorac Oncol 2012;7:825-32.

11. Hellmann MD, Chaft JE, William WN Jr, et al. Pathological response after neoadjuvant chemotherapy in resectable nonsmall-cell lung cancers: proposal for the use of major pathological response as a surrogate endpoint. Lancet Oncol 2014;15:e42-e50.

12. Vokes EE, Ready N, Felip E, et al. Nivolumab versus docetaxel in previously treated advanced non-small-cell lung cancer (CheckMate 017 and CheckMate 057): 3-year update and outcomes in patients with liver metastases. Ann Oncol 2018;29:959-65.

13. Garon EB, Hellmann MD, Rizvi NA, et al. Five-Year Overall Survival for Patients With Advanced NonSmallCell Lung Cancer Treated With Pembrolizumab: Results From the Phase I KEYNOTE-001 Study. J Clin Oncol 2019;37:2518-27.

14. Reck M, Rodriguez-Abreu D, Robinson AG, et al. Pembrolizumab versus Chemotherapy for PD-L1Positive Non-Small-Cell Lung Cancer. N Engl J Med 2016;375:1823-33.

15. Schmid P, Adams S, Rugo HS, et al. Atezolizumab and Nab-Paclitaxel in Advanced Triple-Negative Breast Cancer. N Engl J Med 2018;379:2108-21.

16. Balar AV, Castellano D, O'Donnell PH, et al. First-line pembrolizumab in cisplatin-ineligible patients with locally advanced and unresectable or metastatic urothelial cancer (KEYNOTE-052): A multicentre, single-arm, phase 2 study. Lancet Oncol 2017;18:1483-92.

17. Zhu J, Zhang T, Li J, et al. Association Between Tumor Mutation Burden (TMB) and Outcomes of Cancer Patients Treated With PD-1/PD-L1 Inhibitions: A MetaAnalysis. Front. Pharmacol 2019;10:673.

18. Massard C, Michiels S, Ferte C, et al. High-Throughput Genomics and Clinical Outcome in Hard-to-Treat Advanced Cancers: Results of the MOSCATO 01 Trial. Cancer Discov 2017;7:586-95.

19. Wang W, Yu D, Sarnaik AA, et al. Biomarkers on melanoma patient $\mathrm{T}$ cells associated with ipilimumab treatment. J Transl Med 2012;10:146.

20. Tietze JK, Angelova D, Heppt MV, et al. Low baseline levels of NK cells may predict a positive response to ipilimumab in melanoma therapy. Exp Dermatol 2017;26:622-9.

21. Ji RR, Chasalow SD, Wang L, et al. An immuneactive tumor microenvironment favors clinical response to ipilimumab. Cancer Immunol Immunother 2012;61:1019-31.

22. Sakai H, Kokura S, Ishikawa T, et al. Effects of anticancer agents on cell viability, proliferative activity and cytokine production of peripheral blood mononuclear cells. J Clin Biochem Nutr 2013;52:64-71.

23. Pol J, Vacchelli E, Aranda F, et al. Trial Watch: Immunogenic cell death inducers for anticancer chemotherapy. Oncoimmunology 2015;4:e1008866.

24. Goldberg MV, Maris CH, Hipkiss EL, et al. Role of PD-1 and its ligand, B7-H1, in early fate decisions of CD8 T 
cells. Blood 2007;110:186-92.

25. Liu J, Blake SJ, Yong MC, et al. Improved efficacy of neoadjuvant compared to adjuvant immunotherapy to eradicate metastatic disease. Cancer Discov 2016;6:1382-99.

Cite this article as: Chen Y, Yan B, Xu F, Hui Z, Zhao G, Liu J, Zhang H, Zeng Z, Zhang R, Provencio M, Ren X, You J. Neoadjuvant chemoimmunotherapy in resectable stage IIIA/ IIIB non-small cell lung cancer. Transl Lung Cancer Res 2021;10(5):2193-2204. doi: 10.21037/tlcr-21-329
26. Salmon H, Idoyaga J, Rahman A, et al. Expansion and activation of CD103(+) dendritic cell progenitors at the tumor site enhances tumor responses to therapeutic PDL1 and BRAF inhibition. Immunity 2016;44:924-38. 


\section{tumor extracavity type tumor intracavity type}

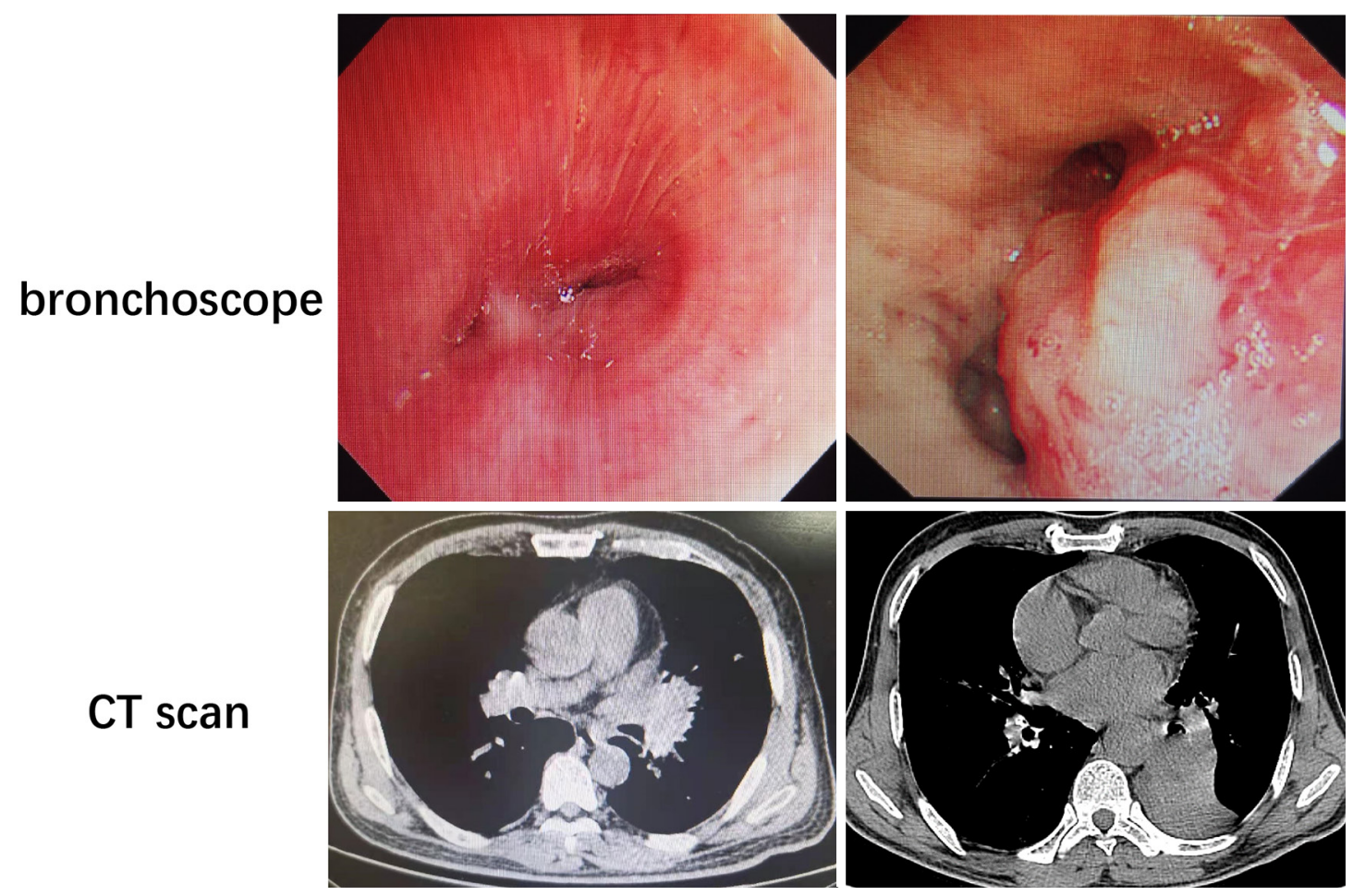

Figure S1 Representative images of bronchoscopy and CT scan of tumor intracavitary or extracavitary type. For the intracavitary type, bronchoscopy shows protrusive growth of intrabronchial masses and obstruction of the bronchus and CT images shows distal fan-shaped atelectasis. And for the extracavitary type, bronchoscope shows bronchial mucosa redness, swelling, shrinkage, and CT image shows central bronchial masses. 